\title{
Hypercoagulation and Antithrombotic Treatment in Coronavirus 2019: A New Challenge
}

\author{
Francesco Violi ${ }^{1}$ Daniele Pastori ${ }^{1}$ Roberto Cangemi ${ }^{2}$ Pasquale Pignatelli ${ }^{1}$ Lorenzo Loffredo ${ }^{10}$ \\ ${ }^{1}$ Department of Clinical, Internal Medicine, Anesthesiologic and \\ Cardiovascular Sciences, Sapienza University of Rome, Italy \\ 2 Department of Translational and Precision Medicine, Sapienza \\ University of Rome, Italy \\ Address for correspondence Francesco Violi, MD, I Clinica Medica, \\ Sapienza University of Rome, Viale del Policlinico 155, Rome 00161, \\ Italy (e-mail: francesco.violi@uniroma1.it).
}

Thromb Haemost 2020;120:949-956.

\begin{abstract}
Keywords

- thrombosis

- hypercoagulability

- infectious diseases

The novel coronavirus 2019 (COVID-19) is clinically characterized by severe acute respiratory syndrome coronavirus 2 (SARS-CoV-2), which is responsible for a high number of patients needing mechanical ventilation or intensive care units treatment and for the elevated mortality risk. A link between COVID-19 and multiorgan failure may be dependent on the fact that most COVID-19 patients are complicated by pneumonia, which is known to be associated with early changes of clotting and platelet activation and artery dysfunction; these changes may implicate in thrombotic-related events such as myocardial infarction and ischemic stroke. Recent data showed that myocardial injury compatible with coronary ischemia may be detectable in SARS-CoV-2 patients and laboratory data exploring clotting system suggest the presence of a hypercoagulation state. Thus, we performed a systematic review of COVID-19 literature reporting measures of clotting activation to assess if changes are detectable in this setting and their relationship with clinical severity. Furthermore, we discussed the biologic plausibility of the thrombotic risk in SARS-CoV-2 and the potential use of an antithrombotic treatment.
\end{abstract}

\section{Introduction}

The novel coronavirus 2019(COVID-19) is being rapidly diffused worldwide from China to Europe and now in United States, so creating serious medical and social issue to contain its potentially dangerous complications. COVID-19 is, in fact, complicated by severe acute respiratory syndrome coronavirus 2 (SARS-CoV-2), which is responsible for a high number of patients needing mechanical ventilation or intensive care units (ICU) treatment and for the elevated mortality risk. However, lung damage is not the only reason for the high death risk as other organs such as heart and kidney may be seriously damaged and favor poor survival. This entails that SARS-CoV2 is a multiorgan disease with mechanisms such as, for example, systemic inflammation which may precipitate organ failure. A link between COVID-19 and multiorgan failure may be dependent on the fact that almost all patients hospitalized for COVID-19 disease are complicated by pneumonia, which is known to be associated with systemic inflammation and thrombotic-related events such as myocardial infarction (MI) and ischemic stroke. ${ }^{1,2}$ Accordingly, previous studies reported that patients with pneumonia display changes of clotting and platelet activation, ${ }^{3,4}$ which occur during the early phase of disease and may precipitate systemic or local thrombosis. It is arguable, therefore, that SARS-CoV-2 patients may experience thrombotic events in coronary and cerebral trees or in other circulatory districts including lung and kidney. Clinical data so far reported are suggestive of vascular complications occurring in the coronary circulation while the involvement of other districts is still unclear. ${ }^{5-7}$ Conversely, there are many studies reporting that the behavior of clotting variables and platelet count in COVID-19 patients is suggestive of a hypercoagulation received

April 1, 2020

accepted

April 13, 2020 (c) 2020 Georg Thieme Verlag KG Stuttgart · New York
DOI https://doi.org/ 10.1055/s-0040-1710317. ISSN 0340-6245. 
state. As clinical presentation and complications of COVID-19 is wide, in this review we sought to determine if changes of clotting variables are dependent on the severity of the disease. To this purpose, we performed a systematic review of the SARSCoV-2 literature reporting measures of clotting activation to assess if changes are detectable in this setting and their relationship with clinical severity. Thus, clotting variables have been examined in patients divided according to the severity of SARSCoV-2. Furthermore, we discussed the biologic plausibility of the thrombotic risk in SARS-CoV-2 and the potential use of an antithrombotic treatment.

\section{Eligibility Criteria}

Types of studies: clinical studies in patients with COVID-19 infection in humans that assessed the following laboratory parameters: d-dimer, platelet counts, prothrombin time, activated partial thromboplastin time, alanine aminotransferase, and aspartate aminotransferase. The studies included in this systematic review had to show COVID-19 infection with severe disease and control group.

No publication date or publication status restrictions were imposed. Only publications written in English language were included in this systematic review.

\section{Information Sources}

The studies were identified by searching electronic databases. This search was applied to PubMed, ISI Web of Science, SCOPUS, and Cochrane database. The last search was run on March 28, 2020. Reference lists of all studies included in the present systematic review were screened for potential additional eligible studies.

\section{Search}

Two investigators (D.P. and L.L.) independently searched in the electronic databases combining the following text terms and MeSH terms: (("coronavirus" [MeSH Terms] OR "coronavirus" [All Fields]) AND ("COVID-19" [All Fields] OR "COVID-2019" [All Fields] OR "severe acute respiratory syndrome coronavirus 2"[Supplementary Concept] OR "severe acute respiratory syndrome coronavirus 2"[All Fields] OR "2019-nCoV"[All Fields] OR "SARS-CoV-2"[All Fields] OR "2019nCoV"[All Fields] OR (“"Wuhan"[All Fields] AND ("coronavirus"[MeSH Terms] OR “coronavirus"[All Fields])) AND (2019/12[PDAT] OR 2020 [PDAT])))) AND “humans"[MeSH Terms].

\section{Study Selection}

Two authors (L.L. and D.P.) independently reviewed titles and abstracts generated by search. Studies were excluded if the title and/or abstract showed that the papers did not meet the selection criteria of our systematic review. For potentially eligible studies or if the relevance of an article could not be excluded with certitude, we procured the full text. Disagreements were resolved by discussion between L.L. and D.P.; if no agreement reached, a third author (F.V.) decided.

Studies not including a control group and animal studies were excluded. Case reports, editorials, commentaries, letters, review articles, and guidelines were also excluded from the analysis. -Fig. 1 reports the PRISMA diagram.

\section{Clotting Changes in Coronavirus 2019}

We analyzed some variables of variable of clotting and fibrinolysis activation along with platelet count in all COVID-19 population ( - Table $\mathbf{1}$ ) and in patients according to disease severity ( - Table 2 ). The latter was defined as acute respiratory distress syndrome (ARDS), need of mechanical ventilation/ICU treatment, nonsurvivor patients, or severe pneumonia. ${ }^{8}$ Our analysis considered globally nine reports. ${ }^{9-17}$

\section{D-Dimer}

D-dimers values were reported in all (9-12 and 14-17) but one study ${ }^{13}$ and definition of increased values in six out of eight studies. $.10,12,15-17$ Among the six studies, one ${ }^{17}$ reported only the percentage of patients with increased values of D-dimer while the other reported also the absolute values. The percentage of patients with elevated D-dimer was almost wide with high values ranging from 14 to $46 \%$. Analysis of D-dimer according to disease severity was reported in six studies (-Table 2), and all consistently showed increased values of D-dimer in patients with severe versus nonsevere disease; significance was shown in all but two studies. ${ }^{14,17}$ These data are in accordance with a recent meta-analysis showing enhanced D-dimer according to disease severity. ${ }^{18}$

\section{Platelet Count}

Platelet count data were extrapolated from seven studies, ${ }^{9-13,16,17}$ of which six $^{9-12,16,17}$ reported changes in terms of low platelet count. Reduced platelet count was detected from 5 to $18 \%$ of the entire population; platelet count was roughly around $100,000 \mu \mathrm{L}$ and in some cases below this value. The impact of disease severity on platelet count was reported in five studies ${ }^{11-13,16,17}$ with a significant reduction in nonsurvivors compared with survivors; however, a trend toward a reduction of platelet count in patients with severe disease was reported in all studies, which is in accordance with a previous report showing that thrombocytopenia is more frequent in patients with severe disease. $^{19}$

\section{Prothrombin Time}

We extrapolated data from 8 (9-16) studies, but only $5^{9,10,12,15,16}$ reported changes in terms of PT prolongation, which was detected from 2 to $11 \%$ of the entire population. The rate of PT prolongation was not reported in all, but one study $^{9}$ where a small prolongation can be appreciated. Conversely, analysis of PT according to disease severity was done in six studies, ${ }^{11-16}$ where PT was reported be longer in patients with severe compared with those without severe disease; prolongation of PT was more evident in nonsurvivors with values exceeding 15 to 16 seconds.

\section{Activated Partial Thromboplastin Time}

We extrapolated data from six studies (9-11 and 14-16) but only three ${ }^{9,10,16}$ reported changes in terms of aPTT prolongation, which was detected from 6 to $26 \%$ of the entire population. The rate of aPTT prolongation was unclear with only one study showing a modest increase. Five studies reported the behavior 

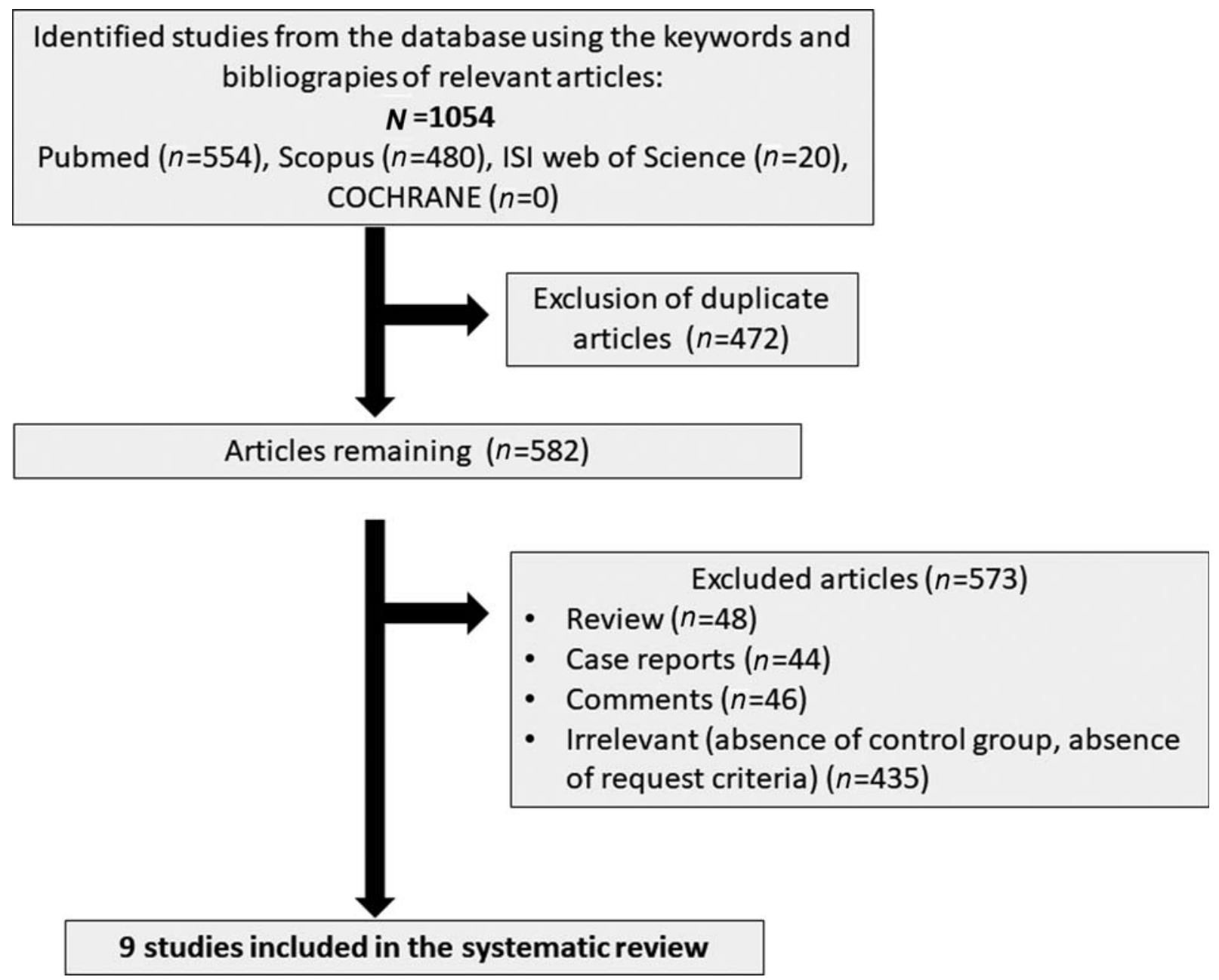

Fig. 1 Flow chart of the studies included in the systematic review.

of aPTT according to the disease severity. ${ }^{11,12,14-16}$ Data are difficult to interpret as prolongation as well as shortening aPTT were reported in patients with severe versus nonsevere disease; prolongation of aPTT seemed to occur in nonsurvivors, but data need to be confirmed.

\section{Liver Failure in Coronavirus 2019}

As the prolongation of PT and aPTT could be dependent upon impaired liver biosynthesis and/or coagulopathy, ${ }^{20}$ we analyzed if COVID-19 patients showed changes in transaminases.

We found six studies ${ }^{9-12,16,17}$ reporting alanine transaminase (ALT) serum values: changes in terms of values exceeding normal range was reported in four, ${ }^{9,10,12,17}$ which showed ALT increase in 12 to $31 \%$ of patients. The rate of ALT increase was unclear as only one study showed absolute values, which were approximately twofold the normal range. Four studies ${ }^{11,12,16,17}$ reported ALT values according to the disease severity, and only two showed a modest increase in case of ARDS or poor survival. ${ }^{12,16}$

We found five studies ${ }^{9-11,16,17}$ reporting aspartate aminotransferase (AST) serum values; changes in terms of values exceeding normal range were reported in four, ${ }^{9-11,17}$ which showed AST increase in 18 to $62 \%$ of the entire population and in one study reporting absolute values, the AST increase was modest. Three studies ${ }^{11,16,17}$ reported AST values according to the disease severity and only two ${ }^{11,16}$ showed a modest but significant increase (-Table 2). Together, these data show that in COVID-19 liver failure is almost modest and, thereby, does not seem to have an impact on clotting changes.

\section{Biological Plausibility of Hypercoagulation in Coronavirus 2019}

Hospitalized patients affected by COVID-19 are frequently complicated by serious pneumonia, which may occur, from roughly 80 to $100 \%$ of cases. ${ }^{11,21,22}$ Considering that respiratory tract infections are associated with an increased risk of vascular disease, including artery and venous thrombosis, it is not surprising that SARS-CoV-2 may be complicated by clotting changes that ultimately lead to thrombosis. Among artery thrombosis complications patients with pneumonia may experience $\mathrm{MI}$ in approximately $10 \%$ of cases, and less frequently, ischemic stroke. ${ }^{3}$ In SARS-CoV-2 patients, detailed description of cardiovascular complications is still lacking even if in some reports it is possible to appreciate data indicating the occurrence of myocardial damage as depicted by enhanced levels of troponin associated or less with EKG changes compatible with MI. ${ }^{6,7}$ Conversely, no data have been so far reported regarding 


\begin{tabular}{|c|c|c|c|c|c|c|c|c|c|c|c|c|c|c|c|c|c|c|c|}
\hline 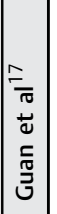 & $\begin{array}{l} \\
\text { gे } \\
\circ \\
-\end{array}$ & & $\overrightarrow{\bar{E}}$ & 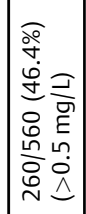 & 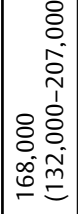 & $\begin{array}{l}\vec{D} \\
\stackrel{0}{\circ} \\
x\end{array}$ & 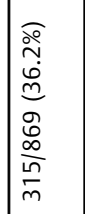 & & & & & & & & $\vec{\partial}$ & 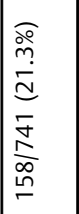 & & $\vec{\partial}$ & 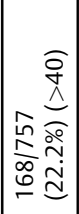 \\
\hline 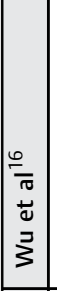 & $\bar{\sim}$ & 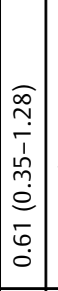 & 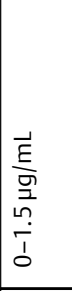 & 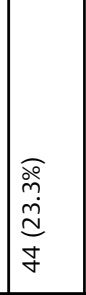 & 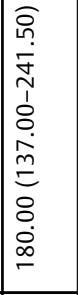 & 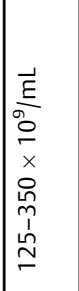 & 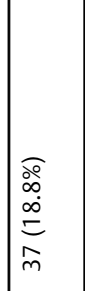 & 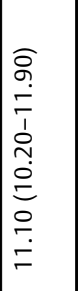 & 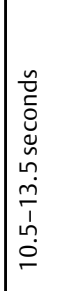 & $\begin{array}{l}\bar{d} \\
\stackrel{d}{d} \\
\stackrel{d}{d}\end{array}$ & 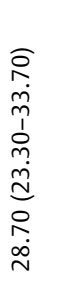 & 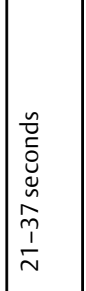 & 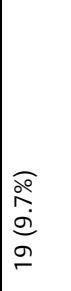 & 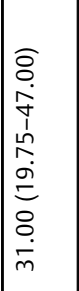 & $\begin{array}{l}\vec{\partial} \\
0 \\
0 \\
h \\
\alpha \\
\sigma\end{array}$ & & 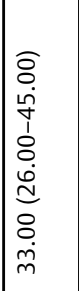 & 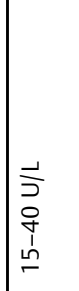 & \\
\hline 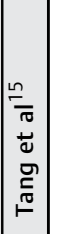 & $\stackrel{\infty}{-}$ & 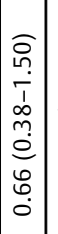 & 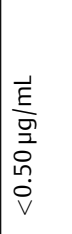 & 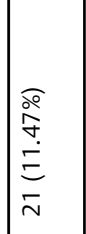 & & $\begin{array}{l}\overrightarrow{2} \\
\frac{0}{0} \\
x\end{array}$ & 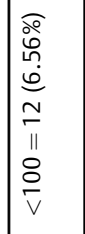 & 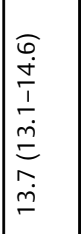 & 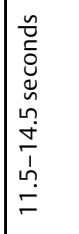 & 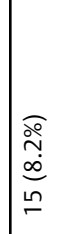 & 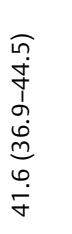 & 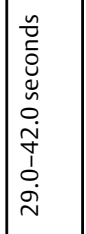 & & & & & & & \\
\hline 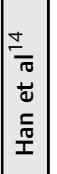 & मे & 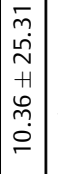 & $\frac{\vec{J}}{\mathrm{G}}$ & & & & & 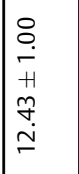 & $\breve{\varpi}$ & & 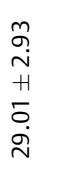 & 岕 & & & & & & & \\
\hline 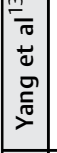 & in & & & & 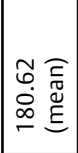 & $\begin{array}{l}\frac{2}{2} \\
\frac{0}{x} \\
x\end{array}$ & & 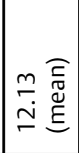 & 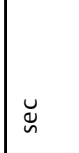 & & & & & & & & & & \\
\hline 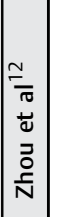 & $\bar{\sigma}$ & \begin{tabular}{|c|}
$\widehat{\tilde{r}}$ \\
$\tilde{p}$ \\
$\dot{1}$ \\
$\dot{0}$ \\
0 \\
0 \\
0 \\
0
\end{tabular} & 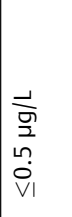 & 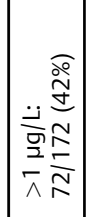 & 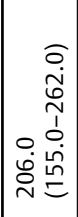 & $\begin{array}{l}\vec{\partial} \\
\text { ने } \\
\stackrel{0}{x} \\
x\end{array}$ & 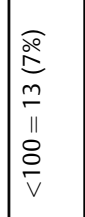 & 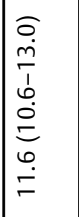 & 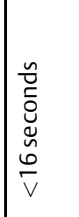 & 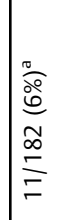 & & & 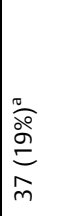 & 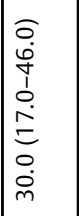 & $\vec{\partial}$ & 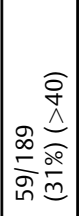 & & & \\
\hline 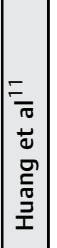 & $\bar{\sigma}$ & $\begin{array}{c}\widehat{c} \\
m \\
i \\
1 \\
m \\
e \\
n \\
0 \\
0\end{array}$ & $\vec{\sigma}$ & & 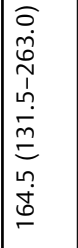 & $\begin{array}{l}\frac{\vec{\partial}}{\circ} \\
\frac{0}{x}\end{array}$ & 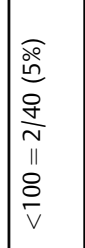 & 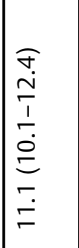 & 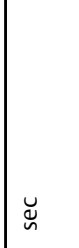 & & 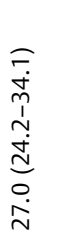 & 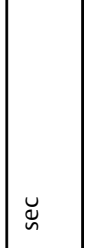 & & 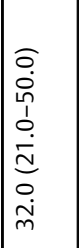 & $\vec{\partial}$ & & 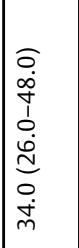 & $\vec{\partial}$ & 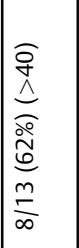 \\
\hline 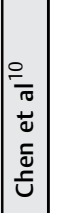 & த & 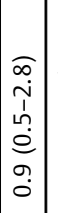 & 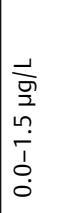 & 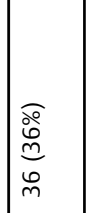 & 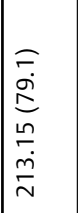 & 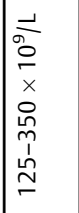 & 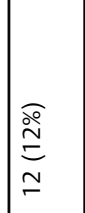 & 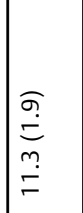 & 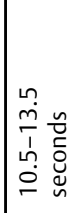 & 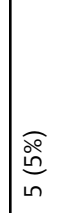 & 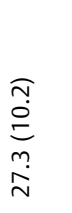 & 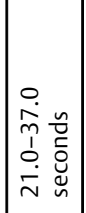 & $\stackrel{\overparen{8}}{\stackrel{80}{0}}$ & 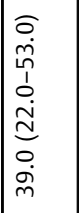 & 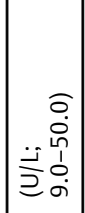 & 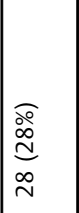 & $\mid \begin{array}{l}\stackrel{0}{0} \\
\dot{m}\end{array}$ & 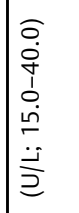 & 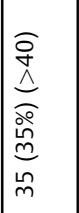 \\
\hline 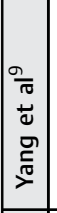 & $\stackrel{g}{\square}$ & 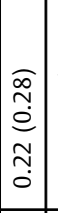 & 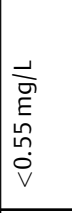 & 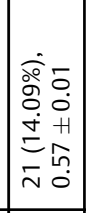 & 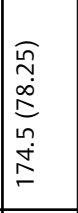 & 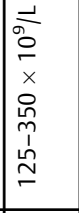 & 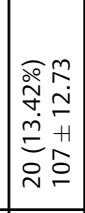 & 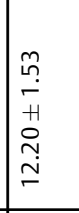 & 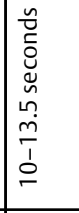 & 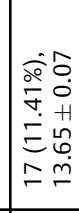 & $\begin{array}{l}\infty \\
\stackrel{\infty}{+} \\
\dot{+} \\
+ \\
\stackrel{2}{N} \\
\underset{m}{m}\end{array}$ & 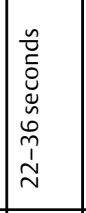 & 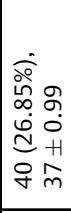 & 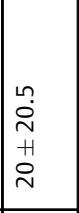 & 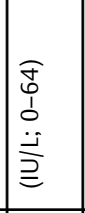 & 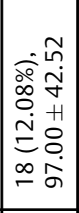 & 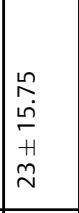 & $\begin{array}{l}\widehat{o} \\
\dot{y} \\
0 \\
\ddot{\vec{j}} \\
\underline{ٍ}\end{array}$ & 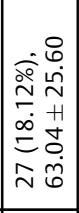 \\
\hline & 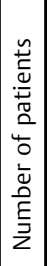 & 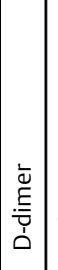 & 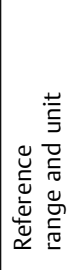 & 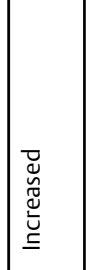 & 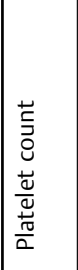 & 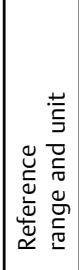 & 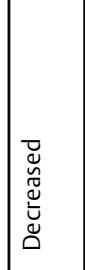 & 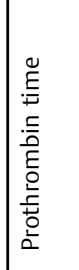 & 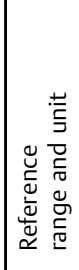 & 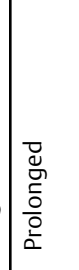 & 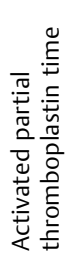 & 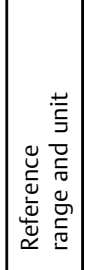 & 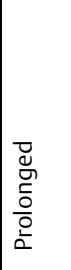 & 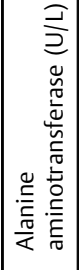 & 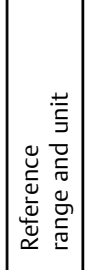 & 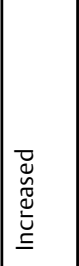 & 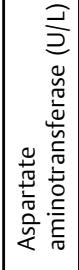 & 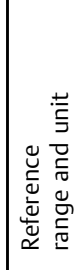 & 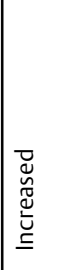 \\
\hline
\end{tabular}


Table 2 Coagulation and liver parameters in patients with coronavirus 2019 infection according to severity of the disease

\begin{tabular}{|c|c|c|c|c|}
\hline Study (year) & Number of patients & Severe & Nonsevere & $p$-Value \\
\hline \multicolumn{5}{|l|}{ D-dimer } \\
\hline Huang et $\mathrm{al}^{11}(2020)$ & 41 (13 ICU, 28 no ICU) & $2.4(0.6-14.4)$ & $0.5(0.3-0.8)$ & $p=0.0042$ \\
\hline \multirow[t]{2}{*}{ Zhou et al ${ }^{12}(2020)$} & 191 (54 nonsurvivors, 137 survivors) & $5.2(1.5-21.1)^{c}$ & $0.6(0.3-1.0)^{c}$ & $p<0.0001$ \\
\hline & & $>1 \mu \mathrm{g} / \mathrm{L}=44(81 \%)$ & $>1 \mu \mathrm{g} / \mathrm{L}=28 / 118(24 \%)$ & $p<0.0001$ \\
\hline Han et $\mathrm{al}^{14}(2020)$ & 94 (49 ordinary, 35 severe, 10 critical) & $\begin{array}{l}\text { Severe: } 19.11 \pm 35.48 \\
\text { Critical: } 20.04 \pm 32.39\end{array}$ & $2.14 \pm 2.88$ & n.r. \\
\hline Tang et al ${ }^{15}(2020)$ & 183 (21 nonsurvivors, 162 survivors) & $2.12(0.77-5.27)$ & $0.61(0.35-1.29)$ & $p<0.001$ \\
\hline \multirow[t]{2}{*}{ Wu et $\mathrm{al}^{16, \mathrm{a}}(2020)$} & 117 (no ARDS, 84 ARDS) & $1.16(0.46-5.37)$ & $0.52(0.33-0.93)$ & $p<0.001$ \\
\hline & 40 ARDS alive, 44 ARDS died & $3.95(1.15-10.96)$ & $0.49(0.31-1.18)$ & $p=0.001$ \\
\hline Guan et al ${ }^{17}(2020)$ & $\begin{array}{l}\text { 1,099 COVID-19 patients (926 non- } \\
\text { severe vs. } 173 \text { severe) }\end{array}$ & $65 / 109(59.6)>0.5 \mathrm{mg} / \mathrm{L}$ & $195 / 451(43.2) \mathrm{mg} / \mathrm{L}$ & n.r. \\
\hline \multicolumn{5}{|l|}{ Platelet count } \\
\hline \multirow[t]{2}{*}{ Huang et $\mathrm{al}^{11}(2020)$} & 41 (13 ICU, 28 no ICU) & $196(165-263)$ & $149(131-263)$ & $p=0.450$ \\
\hline & & $<100=1 / 13(8 \%)$ & $<100=1 / 27(4 \%)$ & $p=0.450$ \\
\hline \multirow[t]{2}{*}{ Zhou et al ${ }^{12}(2020)$} & 191 (54 nonsurvivors, 137 survivors) & $165.5(107.0-229.0)$ & $220.0(168.0-271.0)$ & $p<0.0001$ \\
\hline & & $<100=11(20 \%)$ & $<100=2(1 \%)$ & $p<0.0001$ \\
\hline Yang et al ${ }^{13}(2020)$ & 52 (32 nonsurvivors, 20 survivors) & $191(63)$ & $164(74)$ & n.r. \\
\hline \multirow[t]{2}{*}{ Wu et a ${ }^{16}(2020)$} & 117 (no ARDS, 84 ARDS) & $187.00(124.50-252.50)$ & $178.00(140.00-239.50)$ & 0.730 \\
\hline & 40 ARDS alive, 44 ARDS died & $162.00(110.50-231.00)$ & $204.00(137.25-262.75)$ & 0.100 \\
\hline \multirow[t]{2}{*}{ Guan et al ${ }^{17}(2020)$} & $\begin{array}{l}\text { 1,099 COVID-19 patients (926 non- } \\
\text { severe vs. } 173 \text { severe) }\end{array}$ & $\begin{array}{l}137,500 \\
(99,000-179,500)\end{array}$ & $\begin{array}{l}172,000 \\
(139,000-212,000)\end{array}$ & n.r. \\
\hline & $<150,000$ per $\mathrm{mm}^{3}$ & $90 / 156(57.7)$ & $225 / 713(31.6)$ & n.r. \\
\hline \multicolumn{5}{|l|}{ Prothrombin time } \\
\hline Huang et al ${ }^{11}$ & 41 (13 ICU, 28 no ICU) & $12.2(11.2-13.4)$ & $10.7(9.8-12.1)$ & $p=0.012$ \\
\hline \multirow[t]{2}{*}{ Zhou et al ${ }^{12}(2020)$} & 191 (54 nonsurvivors, 137 survivors) & $12.1(11.2-13.7)$ & $11.4(10.4-12.6)$ & $p=0.0004$ \\
\hline & & $\geq 16$ seconds $=7(13 \%)$ & $\geq 16$ seconds $=4 / 128(3 \%)$ & $p=0.016$ \\
\hline Yang et al ${ }^{13}$ & 52 (32 nonsurvivors, 20 survivors) & $12.9(2.9)$ & $10.9(2.7)$ & n.r. \\
\hline Han et $\mathrm{al}^{14}(2020)$ & 94 (49 ordinary, 35 severe, 10 critical) & $\begin{array}{l}\text { Severe: } 12.65 \pm 1.13 \\
\text { Critical: } 12.80 \pm 0.87\end{array}$ & $12.20 \pm 0.88$ & n.r. \\
\hline Tang et al ${ }^{15}(2020)$ & 183 (21 nonsurvivors, 162 survivors) & $15.5(14.4-16.3)$ & $13.6(13.0-14.3)$ & $p<0.001$ \\
\hline \multirow[t]{2}{*}{ Wu et a ${ }^{16}(2020)$} & 117 no ARDS, 84 ARDS & $11.70(11.10-12.45)$ & $10.60(10.10-11.50)$ & $p<0.001$ \\
\hline & 40 ARDS alive, 44 ARDS died & $11.60(11.10-12.45)$ & $11.75(10.95-12.45)$ & $p=0.870$ \\
\hline \multicolumn{5}{|c|}{ Activated partial thromboplastin time } \\
\hline Huang et $\mathrm{al}^{11}$ & 41 (13 ICU, 28 no ICU) & $26.2(22.5-33.9)$ & $27.7(24.8-34.1)$ & $p=0.570$ \\
\hline Zhou et al ${ }^{12}(2020)$ & 191 (54 nonsurvivors, 137 survivors) & $27(50 \%)^{\mathrm{b}}$ & $10(7 \%)^{b}$ & $p<0.0001$ \\
\hline Han et $\mathrm{al}^{14}(2020)$ & 94 (49 ordinary, 35 severe, 10 critical) & $\begin{array}{l}\text { Severe: } 29.53 \pm 3.48 \\
\text { Critical: } 29.41 \pm 1.68\end{array}$ & $28.56 \pm 2.66$ & n.r. \\
\hline Tang et al ${ }^{15}(2020)$ & 183 (21 nonsurvivors, 162 survivors) & $44.8(40.2-51.0)$ & $41.2(36.9-44.0)$ & $p=0.096$ \\
\hline \multirow[t]{2}{*}{ Wu et al ${ }^{16}(2020)$} & 117 (no ARDS, 84 ARDS) & $26.00(22.55-35.00)$ & $29.75(25.55-32.85)$ & $p=0.130$ \\
\hline & 40 ARDS alive, 44 ARDS died & $24.10(22.25-28.35)$ & $29.60(24.00-35.75)$ & $p=0.040$ \\
\hline \multicolumn{5}{|l|}{ ALT } \\
\hline Huang et $\mathrm{al}^{11}(2020)$ & 41 (13 ICU, 28 no ICU) & $49.0(29.0-115.0)$ & $27.0(19.5-40.0)$ & 0.038 \\
\hline \multirow[t]{2}{*}{ Zhou et al ${ }^{12}(2020)$} & 191 (54 nonsurvivors, 137 survivors) & $40.0(24.0-51.0)$ & $27.0(15.0-40.0)$ & 0.005 \\
\hline & $>40$ & $26(48 \%)$ & $33 / 135(24 \%)$ & 0.0015 \\
\hline \multirow[t]{2}{*}{ Wu et $\mathrm{al}^{16}(2020)^{\mathrm{a}}$} & 117 (no ARDS, 84 ARDS) & $35.00(21.50-52.50)$ & $27.00(18.00-41.50)$ & 0.01 \\
\hline & 40 ARDS alive, 44 ARDS died & $39.00(20.50-52.50)$ & $35.00(23.25-55.25)$ & 0.71 \\
\hline Guan et al ${ }^{17}(2020)$ & $\begin{array}{l}\text { 1,099 COVID-19 patients } \\
\text { (926 nonsevere vs. } 173 \text { severe) }\end{array}$ & $38 / 135(28.1)>40 \mathrm{U} / \mathrm{L}$ & $120 / 606(19.8)>40 \mathrm{U} / \mathrm{L}$ & n.r. \\
\hline
\end{tabular}


Table 2 (Continued)

\begin{tabular}{|l|l|l|l|l|}
\hline Study (year) & Number of patients & Severe & Nonsevere & $p$-Value \\
\hline AST & $41(13$ ICU, 28 no ICU) & $44.0(30.0-70.0)$ & $34.0(24.0-40.5)$ & 0.10 \\
\hline Huang et al ${ }^{11}$ & 117 (no ARDS, 84 ARDS) & $38.00(30.50-53.00)$ & $30.00(24.00-38.50)$ & $<0.001$ \\
\hline Wu et al ${ }^{16}(2020)^{\text {a }}$ & 40 ARDS alive, 44 ARDS died & $37.00(30.00-52.00)$ & $38.50(32.25-57.25)$ & 0.21 \\
\hline & $\begin{array}{l}1099 \text { COVID-19 patients }(926 \text { nonse- } \\
\text { vere vs. 173 severe) }\end{array}$ & $56 / 142(39.4)>40$ U/L & $112 / 615(18.2)>40$ U/L & n.r. \\
\hline Guan et al ${ }^{17}(2020)$ & & & \\
\hline
\end{tabular}

Abbreviations: ALT, alanine transaminase; ARDS, acute respiratory distress syndrome; AST, aspartate aminotransferase; $\mathrm{Cl}$, confidence interval; COVID-19, coronavirus 2019; ICU, intensive care unit; n.r., not reported.

${ }^{\mathrm{a}}$ Hazard ratio for progression to ARDS 1.03 (95\% confidence interval: 1.01-1.04), $p<0.001$; hazard ratio for progression to death 1.02 (95\% Cl: $1.01-$ $1.04), p=0.002$.

${ }^{\mathrm{b}}$ Numbers refers to coagulopathy defined as a 3-second extension of prothrombin time or a 5-second extension of activated partial thromboplastin time. ${ }^{c}$ Multivariable odds ratio for in-hospital death for D-dimer $>1 \mu \mathrm{g} / \mathrm{L}=18.42$ (95\% Cl: 2.64-128.55), $p=0.0033$.

deep venous thrombosis. Systemic coagulation abnormalities, including clotting activation and inhibition of anticoagulant factors, have been observed not only in sepsis but also in pneumonia.

At this last regard, we have previously reported that pneumonia may be associated with clotting activation as well as lowering of natural anticoagulants. Thus, we found enhanced levels of the prothrombin fragment $F 1+2$, which is a marker of thrombin generation, along with impaired activation of the anticoagulant Protein $\mathrm{C}$. These changes occurred during the acute phase of the disease and tended to normalize at the discharge. ${ }^{4}$

Platelet activation has been investigated by measuring the soluble levels of CD40L and P-selectin, which were both elevated during the acute phase of pneumonia, along with an overproduction of platelet thromboxane (Tx) $\mathrm{B}^{23}$; of note, platelet TxB2 was independently associated with the occurrence of MI. Finally, we observed that patients with pneumonia display impaired artery dilatation, which is detectable during the acute phase of the disease and improve at discharge. ${ }^{24}$
The mechanism accounting for these changes is not clear, but it is intriguing that Nox2, which is the most important enzyme generating reactive oxidant species (ROS), is overactivated in patients with pneumonia. ${ }^{25}$ Thus, Nox2 is implicated in artery dilatation and platelet activation by inactivating nitric oxide or enhancing platelet eicosanoid production; experimental model of platelet-related thrombosis provided support to the role of Nox2 as in Nox2 animal knockout thrombus growth is prevented. ${ }^{26,27}$

Thus, there are at least three changes occurring during the acute phase of pneumonia, which may precipitate artery and/or venous thrombosis (-Fig. 2). The mechanisms accounting for clotting and vascular changes have not been defined yet by systemic inflammation, including production of reactive oxidant ROS might have a role. In this context, there are growing body of experimental and clinical evidence that Nox2-derived ROS are implicated in both clotting and platelet activation, acting as intrasignaling pathway to promote thrombin generation and platelet aggregation or to impair artery dilatation ${ }^{26,27}$ (-Fig. 2). Data regarding Nox2 in SARS-CoV-2 patients are still lacking but should be

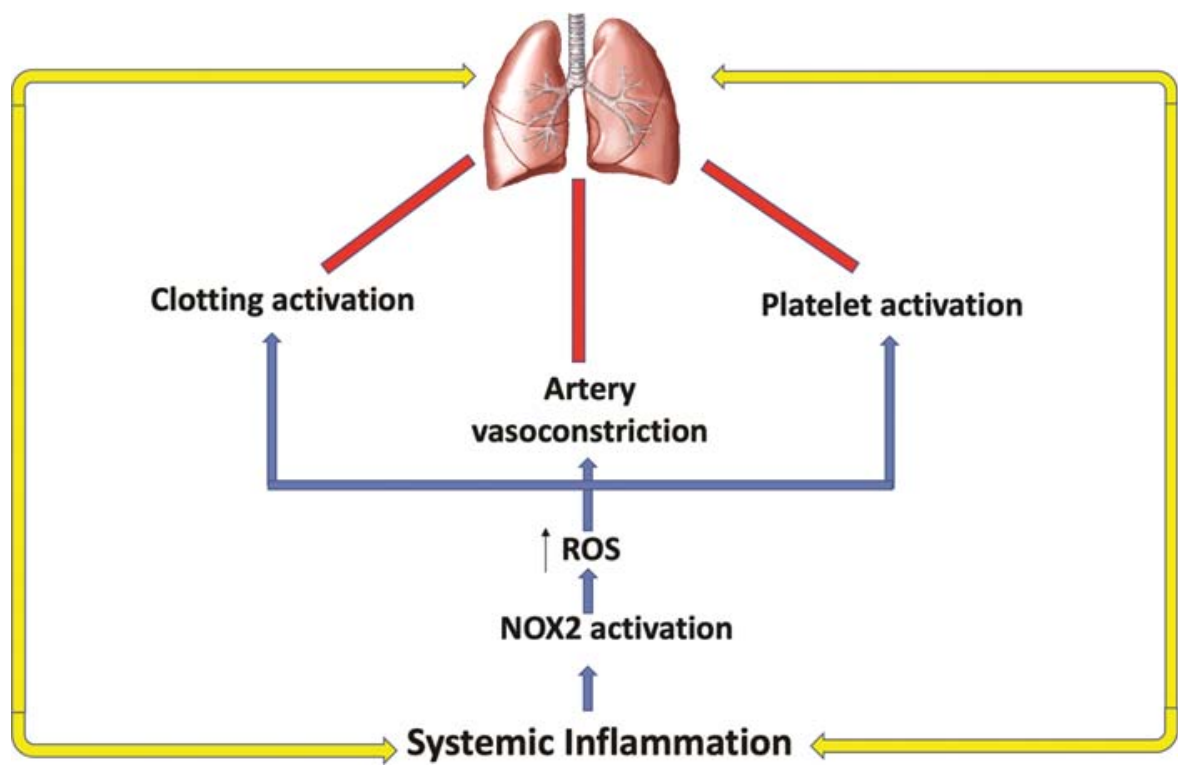

Fig. 2 Hypothetic mechanisms accounting for hypercoagulation in severe acute respiratory syndrome coronavirus 2 patients. 
investigated as experimental data provided evidence for a role played by Nox2 upregulation in the systemic inflammation and pathogenicity elicited by several RNA viruses including influenza virus. ${ }^{28}$

Data regarding clotting changes in SARS-CoV-2 are still sparse and elusive. The only finding which could be consistent with clotting activation is the increase of D-dimer; however, which is not specific for clotting activation as it may also increase as result of systemic proteolysis. ${ }^{29}$ There is also a trend toward PT prolongation and low platelet count in patients with severe disease, suggesting a role for coagulation in precipitating poor outcomes. Preliminary data regarding the relationship between elevated D-dimer and poor outcome would be in favor of this hypothesis, but limited study methodology precludes definite conclusions. ${ }^{12}$

\section{Perspectives and Conclusion}

The data so far reported are suggestive of low-grade intravascular clotting activation, which is evident overall in patients with severe disease. The finding more consistent in support of this hypothesis is the increase of D-dimer, which is almost evident in patients with severe; in a small series of a retrospective study, D-dimer was associated with poor survival. ${ }^{16}$ Concerning PT, aPTT and platelet count data are more elusive even if there is a trend for changes suggestive of a hypercoagulationstate in patients with severe disease. Thus, future study should employ more sophisticated methodology to screen for hypercoagulation state and, in the same time, clarify if platelet activation occurs also in this setting; in this last context, analysis of systemic markers of platelet activation such as soluble P-selectin or IIb/IIla could be useful to address this issue. In the meantime, a hot issue is if SARS-CoV-2 patients should be treated or not with antithrombotic drugs and if the clinical presentation may drive this therapeutic decision (-Fig. 3). On the basis of previous reports showing that patients with pneumonia are associated with platelet and clotting activation, ${ }^{3,4}$ interventional trials aimed at assessing the efficacy of antithrombotic treatment, including aspirin or low-molecular-weight heparin (LMWH), should be planned: preliminary data suggested a potential efficacy of aspirin in patients with pneumonia. ${ }^{30}$ Antithrombotic treatment could be investigated in patients with severe pneumonia or pneumonia complicated by $\mathrm{ARDS}^{31}$ or by sepsis ${ }^{32}$ or needing mechanical ventilation/ICU; the gray area is in case of no pneumonia or pneumonia with low mortality risk according to several risk scores such as $\mathrm{PSI},{ }^{33}$ CURB65, ${ }^{34}$ or SOFA, ${ }^{32}$ in which antithrombotic treatment may be questionable; the coexistence of elevate $\mathrm{D}$-dimer in patients with mild pneumonia may be another setting to be investigated ( - Fig. 3 ).

In absence of clinical trials and mechanism of disease indicating the prominent role, if any, of platelets or clotting system in favoring SARS-CoV-2 sequelae, treating all SARSCoV-2 with prophylactic doses of LMWH, independently from clinical presentation and on the basis of laboratory changes suggestive of a hypercoagulation state, may be intriguing but premature until the interplay between hypercoagulation and SARS- CoV-2 is clarified ${ }^{35}$; previous study in a different clinical setting, that is acutely ill medical patients, demonstrated that without an appropriate definition of hospitalized medical patients at risk of thrombosis, the prophylaxis with LMWH is useless and potentially dangerous. ${ }^{36}$ Thus, patients with pneumonia, particularly those with severe infections as defined by several scores and suffering from complications as ARDS or sepsis or needing mechanical ventilation/ICU treatment could be candidates to

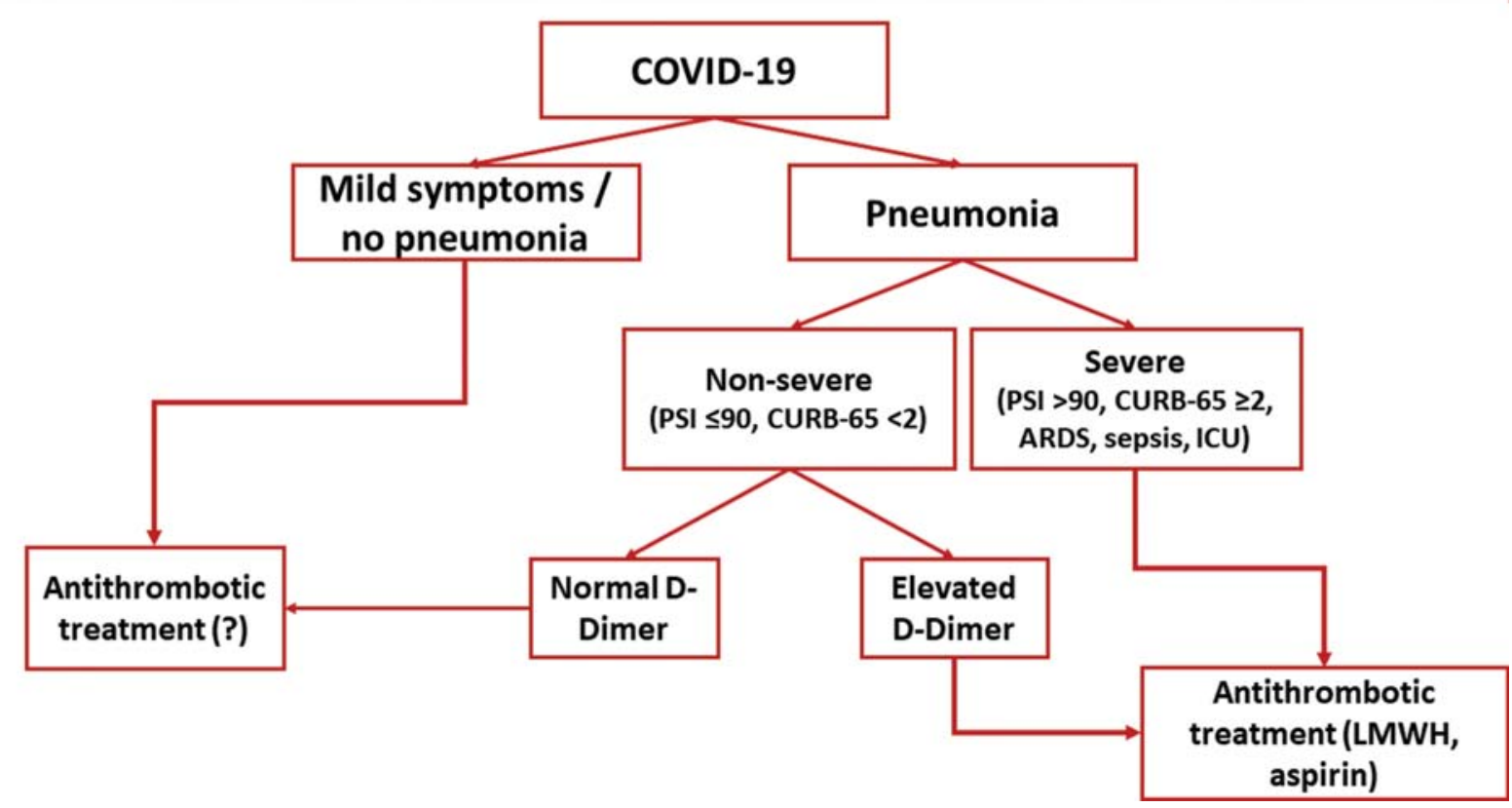

Fig. 3 Suggested algorithm for antithrombotic treatment in severe acute respiratory syndrome coronavirus 2 patients. 
randomized clinical trials with antithrombotic treatment (-Fig. 3) to assess if this approach may blunt SARS-CoV-2 sequelae.

\section{Conflict of Interest}

None declared.

\section{References}

1 Corrales-Medina VF, Musher DM, Shachkina S, Chirinos JA. Acute pneumonia and the cardiovascular system. Lancet 2013;381 (9865):496-505

2 Violi F, Cangemi R, Falcone M, et al; SIXTUS (Thrombosis-Related Extrapulmonary Outcomes in Pneumonia) Study Group. Cardiovascular complications and short-term mortality risk in communityacquired pneumonia. Clin Infect Dis 2017;64(11):1486-1493

3 Cangemi R, Casciaro M, Rossi E, et al; SIXTUS Study Group; SIXTUS Study Group. Platelet activation is associated with myocardial infarction in patients with pneumonia. J Am Coll Cardiol 2014;64 (18):1917-1925

4 Cangemi R, Della Valle P, Calvieri C, et al; SIXTUS Study Group. Low-grade endotoxemia and clotting activation in the early phase of pneumonia. Respirology 2016;21(08):1465-1471

5 Clerkin KJ, Fried JA, Raikhelkar J, et al. Coronavirus disease 2019 (COVID-19) and cardiovascular disease. Circulation 2020. Doi: 10.1161/CIRCULATIONAHA.120.046941

6 Shi S, Qin M, Shen B, et al Association of cardiac injury with mortality in hospitalized patients with COVID-19 in Wuhan, China. JAMA Cardiol 2020. Doi: 10.1001/jamacardio.2020.0950

7 Guo T, Fan Y, Chen M, et al. Cardiovascular implications of fatal outcomes of patients with coronavirus disease 2019 (COVID-19). JAMA Cardiol 2020. Doi: 10.1001/jamacardio.2020.1017

8 Metlay JP, Waterer GW, Long AC, et al. Diagnosis and treatment of adults with community-acquired pneumonia. An Official Clinical Practice Guideline of the American Thoracic Society and Infectious Diseases Society of America. Am J Respir Crit Care Med 2019; 200(07):e45-e67

9 Yang W, Cao Q, Qin L, et al. Clinical characteristics and imaging manifestations of the 2019 novel coronavirus disease (COVID19): a multi-center study in Wenzhou city, Zhejiang, China. J Infect 2020;80(04):388-393

10 Chen N, Zhou M, Dong X, et al. Epidemiological and clinical characteristics of 99 cases of 2019 novel coronavirus pneumonia in Wuhan, China: a descriptive study. Lancet 2020;395(10223):507-513

11 Huang C, Wang Y, Li X, et al. Clinical features of patients infected with 2019 novel coronavirus in Wuhan, China. Lancet 2020;395 (10223):497-506

12 Zhou F, Yu T, Du R, et al. Clinical course and risk factors for mortality of adult inpatients with COVID-19 in Wuhan, China: a retrospective cohort study. Lancet 2020;395(10229):1054-1062

13 Yang X, Yu Y, Xu J, et al. Clinical course and outcomes of critically ill patients with SARS-CoV-2 pneumonia in Wuhan, China: a single-centered, retrospective, observational study. Lancet Respir Med 2020(S2213-2600(20)30079-5):

$14 \mathrm{Han} \mathrm{H}$, Yang L, Liu R, et al. Prominent changes in blood coagulation of patients with SARS-CoV-2 infection. Clin Chem Lab Med 2020. Doi: $10.1515 / \mathrm{cclm}-2020-0188 / 1$

15 Tang N, Li D, Wang X, et al. Abnormal coagulation parameters are associated with poor prognosis in patients with novel coronavirus pneumonia. J Thromb Haemost 2020;18(04):844-847

16 Wu C, Chen X, Cai Y, et al. Risk factors associated with acute respiratory distress syndrome and death in patients with coronavirus disease 2019 pneumonia in Wuhan, China. JAMA Intern Med 2020. Doi: 10.1001/jamainternmed.2020.0994
17 Guan WJ, Liang WH, Zhao Y, et al. China Medical Treatment Expert Group for Covid-19. Comorbidity and its impact on 1590 patients with Covid-19 in China: a nationwide analysis. Eur Respir J 2020: 2000547

18 Lippi G, Favaloro EJ. D-dimer is associated with severity of coronavirus disease 2019: a pooled analysis. Thromb Haemost 2020. Doi: $10.1055 / \mathrm{s}-0040-1709650$

19 Lippi G, Plebani M, Henry BM. Thrombocytopenia is associated with severe coronavirus disease 2019 (COVID-19) infections: a meta-analysis. Clin Chim Acta 2020;506:145-148

20 Violi F, Ferro D. Clotting activation and hyperfibrinolysis in cirrhosis: implication for bleeding and thrombosis. Semin Thromb Hemost 2013;39(04):426-433

21 Wang D, Hu B, Hu C, et al. Clinical characteristics of 138 hospitalized patients with 2019 novel coronavirus-infected pneumonia in Wuhan, China. JAMA. 2020. Doi: 10.1001/jamainternmed.2020.0994

22 Guan WJ, Ni ZY, Hu Y, et al; China Medical Treatment Expert Group for Covid-19. Clinical characteristics of coronavirus disease 2019 in China. N Engl J Med 2020

23 Cangemi R, Pignatelli P, Carnevale R, et al. SIXTUS Study Group. Lowgrade endotoxemia, gut permeability and platelet activation in community-acquired pneumonia. J Infect 2016;73(02):107-114

24 Loffredo L, Cangemi R, Perri L, et al; SIXTUS study group; SIXTUS (thromboSIs-related eXTra-pulmonary oUtcomeS in pneumonia) study group. Impaired flow-mediated dilation in hospitalized patients with community-acquired pneumonia. Eur J Intern Med 2016;36:74-80

25 Violi F, Carnevale R, Calvieri C, et al; SIXTUS study group. Nox2 upregulation is associated with an enhanced risk of atrial fibrillation in patients with pneumonia. Thorax 2015;70(10):961-966

26 Violi F, Pignatelli P. Platelet NOX, a novel target for anti-thrombotic treatment. Thromb Haemost 2014;111(05):817-823

27 Violi F, Carnevale R, Loffredo L, Pignatelli P, Gallin JI. NADPH Oxidase2 and Atherothrombosis: Insight From Chronic Granulomatous Disease. Arterioscler Thromb Vasc Biol 2017;37(02):218-225

28 To EE, Vlahos R, Luong R, et al. Endosomal NOX2 oxidase exacerbates virus pathogenicity and is a target for antiviral therapy. Nat Commun 2017;8(01):69

29 Dempfle CE. Use of D-dimer assays in the diagnosis of venous thrombosis. Semin Thromb Hemost 2000;26(06):631-641

30 Falcone M, Russo A, Cangemi R, et al. Lower mortality rate in elderly patients with community-onset pneumonia on treatment with aspirin. J Am Heart Assoc 2015;4(01):e001595

31 Fan E, Brodie D, Slutsky AS. Acute respiratory distress syndrome: advances in diagnosis and treatment. JAMA 2018;319(07): 698-710

32 Singer M, Deutschman CS, Seymour CW, et al. The third international consensus definitions for sepsis and septic shock (sepsis-3). JAMA 2016;315(08):801-810

33 Aujesky D, Fine MJ. The pneumonia severity index: a decade after the initial derivation and validation. Clin Infect Dis 2008;47 (Suppl 3):S133-S139

34 Myint PK, Kamath AV, Vowler SL, Maisey DN, Harrison BD. The CURB (confusion, urea, respiratory rate and blood pressure) criteria in community-acquired pneumonia (CAP) in hospitalised elderly patients aged 65 years and over: a prospective observational cohort study. Age Ageing 2005;34(01):75-77

35 Tang N, Bai H, Chen X, Gong J, Li D, Sun Z. Anticoagulant treatment is associated with decreased mortality in severe coronavirus disease 2019 patients with coagulopathy. J Thromb Haemost 2020. Doi: $10.1111 /$ jth. 14817

36 Loffredo L, Arienti V, Vidili G, et al. AURELIO Study Group. Low rate of intrahospital deep venous thrombosis in acutely ill medical patients: results from the AURELIO study. Mayo Clin Proc 2019;94 (01):37-43 